\section{En busca de los resultados de la cooperación técnica}

\author{
Lily Jourdan Hidalgo ${ }^{1}$ \\ y Juan Manuel Sotelo ${ }^{1}$
}

La misión de la Organización Panamericana de la Salud (OPS) es brindar cooperación técnica a sus Estados Miembros y estimular ese tipo de cooperación entre ellos para que la población de las Américas conserve un ambiente saludable mientras avanza hacia el desarrollo humano sostenible y alcanza la meta de salud para todos y por todos (1). Se entiende por cooperación técnica aquel proceso mediante el cual los Estados Miembros cooperan con la Organización, como socios iguales, en la definición y logro de sus propias metas de salud y en la promoción de la autosuficiencia en el desarrollo de la salud, por medio de programas que respondan a sus necesidades y prioridades nacionales (2). De acuerdo con esa definición y en la búsqueda permanente de la transparencia, responsabilidad y participación en la actividad institucional, la OPS creó en 1978 el Sistema de Planificación, Programación, Seguimiento y Evaluación de la Región de las Américas (AMPES) para la cooperación técnica en salud (3). El sistema establece los procedimientos de gestión y es una herramienta útil y ágil para tomar decisiones gerenciales.

El AMPES original ha evolucionado, ajustándose a los cambios que afectan a la cooperación técnica en salud en el ámbito internacional y en la OPS en particular. Las modificaciones incluyen una sistematización de la cooperación técnica y el empleo del método denominado "enfoque lógico para la gestión de proyectos" (4), que sirve para estructurar el programa de trabajo de la OPS e identificar los resultados esperados de la cooperación técnica cuando se realizan actividades y se invierten recursos. Además, el enfoque lógico establece una relación causal entre las actividades programadas y los resultados esperados por la OPS, y entre aquellas y el efecto que se espera producir en los países cuando se ejecuta un proyecto.

Durante el último decenio, la OPS ha utilizado la taxonomía de los "enfoques funcionales" (5) para clasificar y agrupar las actividades en categorías que reflejan el propósito de la cooperación técnica: movilización de recursos; diseminación de información; formación de personal; formulación de normas, planes y políticas; fomento de la investigación, y asesoramiento técnico directo. En años recientes, la necesidad de lograr una cooperación más eficaz y transparente, orientándola hacia la obtención de resultados, condujo a la OPS a iniciar un proceso permanente de replanteamiento de la cooperación técnica internacional en salud (RITCH) (6) para

Organización Panamericana de la Salud, Oficina de Análisis y Planificación Estratégica, Washington DC, EUA. Toda correspondencia debe dirigirse a L. Jourdan Hidalgo a la siguiente dirección postal: 525 Twenty-third St., NW, Washington, DC 20037, EUA. 
FIGURA 1. Distribución de recursos que no corresponden a puestos en el BPB de 1998-1999, por enfoques funcionales

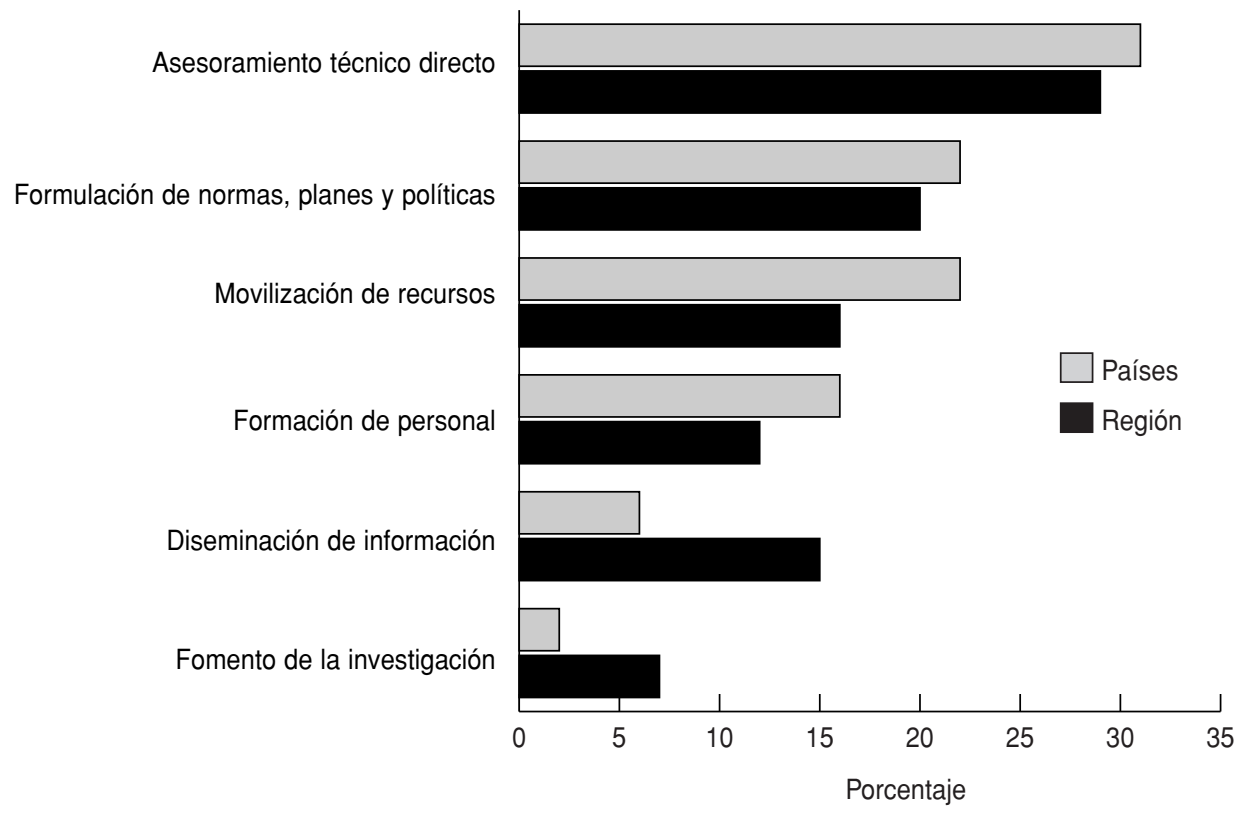

definir este concepto con más claridad. Durante el proceso, se observó que los resultados que la OPS se comprometía a producir mediante la cooperación técnica debían establecerse con más precisión y estar encauzados a responder a las necesidades de los Estados Miembros. Asimismo, se reconoció la importancia de establecer una cultura de programación y evaluación para orientar la definición de los resultados y contribuir a la toma de decisiones, tanto en la OPS como en dichos Estados.

La estructura de los proyectos basada en el enfoque lógico no permitía advertir una relación clara entre el enfoque funcional asignado a las actividades (taxonomía) y los resultados esperados, y por lo tanto se duplicaban las definiciones de dichos enfoques y resultados. No se entendía claramente, por ejemplo, si la capacitación era solo un producto del cual la OPS se hacía responsable (resultado esperado), o si era también un enfoque funcional aplicado a las actividades necesarias para producir otro tipo de resultado esperado. Tampoco se entendía la utilidad de los enfoques funcionales en lo referente a la estructura del programa de trabajo de la cooperación técnica.

Por ejemplo, al examinarse la distribución de recursos del presupuesto bienal por programas (biennial program budget, BPB) de 1998-1999 (7) según los enfoques funcionales, se observó que $60 \%$ de los recursos estaban asignados al asesoramiento técnico directo (figura 1). Sin embargo, cuando se creó la taxonomía de los enfoques funcionales se esperaba que se asignaran menos recursos a ese asesoramiento que a otros enfoques, por tratarse de actividades puntuales que desempeñaban los funcionarios en el área técnica de su especialización en los programas regionales, pero no en las oficinas de la OPS en los países. Por otra parte, al compararse, en términos de enfoques funcionales, la distribución de los recursos programada para 1990 con la programada para 1998-1999, se observó que la formulación de normas, planes y políticas recibió la mayor asignación de recursos y que se incrementaron los recursos asignados al asesoramiento técnico directo (4 puntos porcentuales), a la formación de personal (4 puntos porcentuales) y al fomento de la investigación (5 puntos porcentuales) (figura 2). También se observó que la distribución de recursos según enfoques funcionales en las diferentes unidades subregionales y regionales de la OPS no siempre estaba en consonancia con el tipo de cooperación técnica que aportaban las unidades (figura 3).

\section{ESTUDIO DE LOS ENFOQUES FUNCIONALES}

Entre julio y octubre de 1997, la OPS comenzó un estudio de cuatro fases para examinar la aplica- 


\section{FIGURA 2. Distribución de recursos en programas regionales seleccionados en 1990 y en 1998-1999, por enfoques funcionales}

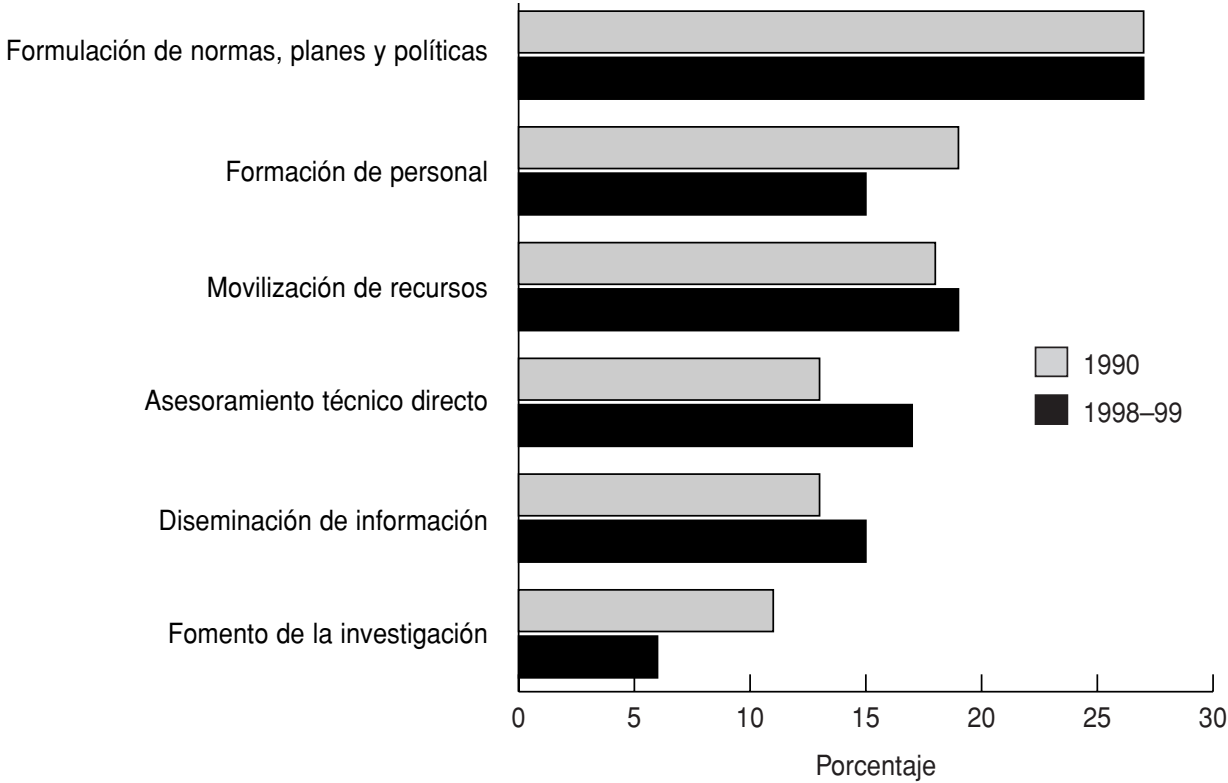

ción de los enfoques funcionales en los proyectos de cooperación técnica. En particular se buscaba determinar su utilidad, su relación con los resultados esperados de los proyectos y su validez para clasificar las actividades de dicha cooperación, así como investigar la necesidad de adoptar enfoques nuevos o de modificar los ya existentes. En el transcurso del estudio se analizó la documentación de siete unidades (cuatro oficinas de país, un centro y dos divisiones) y se utilizaron como material de referencia los presupuestos anuales por programas (annual program budgets, APB) de 1996 y 1997, los informes de la ejecución financiera y programática de los países producidos por el sistema de información de gestión y los informes de la evaluación anual de las unidades del nivel central.

Los objetivos de la primera fase del estudio fueron determinar si los enfoques funcionales eran pertinentes desde el punto de vista de las actividades y si podían utilizarse para definir los resultados esperados de la cooperación técnica descritos en los proyectos. Las conclusiones del análisis ${ }^{2}$ fueron las siguientes:

1. Las unidades tienen dificultad para clasificar las actividades porque los enfoques funcionales no

2 Colaboró en esta fase el consultor Ingvar Ahman. se excluyen mutuamente y las actividades son complejas.

2. Pese a los criterios del enfoque lógico, los resultados esperados descritos no especifican el producto que la Secretaría de la OPS se compromete a entregar en un período determinado ni definen su nivel de responsabilidad.

A continuación, los funcionarios que participaron en el estudio debatieron si los resultados esperados debían clasificarse de acuerdo con los enfoques funcionales del momento o con otros que reflejaran más apropiadamente la responsabilidad directa asumida por la Secretaría. Al final, el grupo propuso una clasificación de resultados esperados sujeta a futuras modificaciones y adaptable a situaciones nuevas o imprevistas. De la nueva clasificación se han eliminado todas las actividades cuyo resultado esperado es producto de procesos administrativos, y no de la cooperación técnica.

\section{Propuesta para la clasificación de los resultados esperados}

1. Redes de cooperación y alianzas

Establecimiento de redes institucionales y programas que intervienen en la cooperación, asî como coaliciones y convenios de cooperación; 
FIGURA 3. Distribución de recursos por enfoques funcionales, 1998-1999

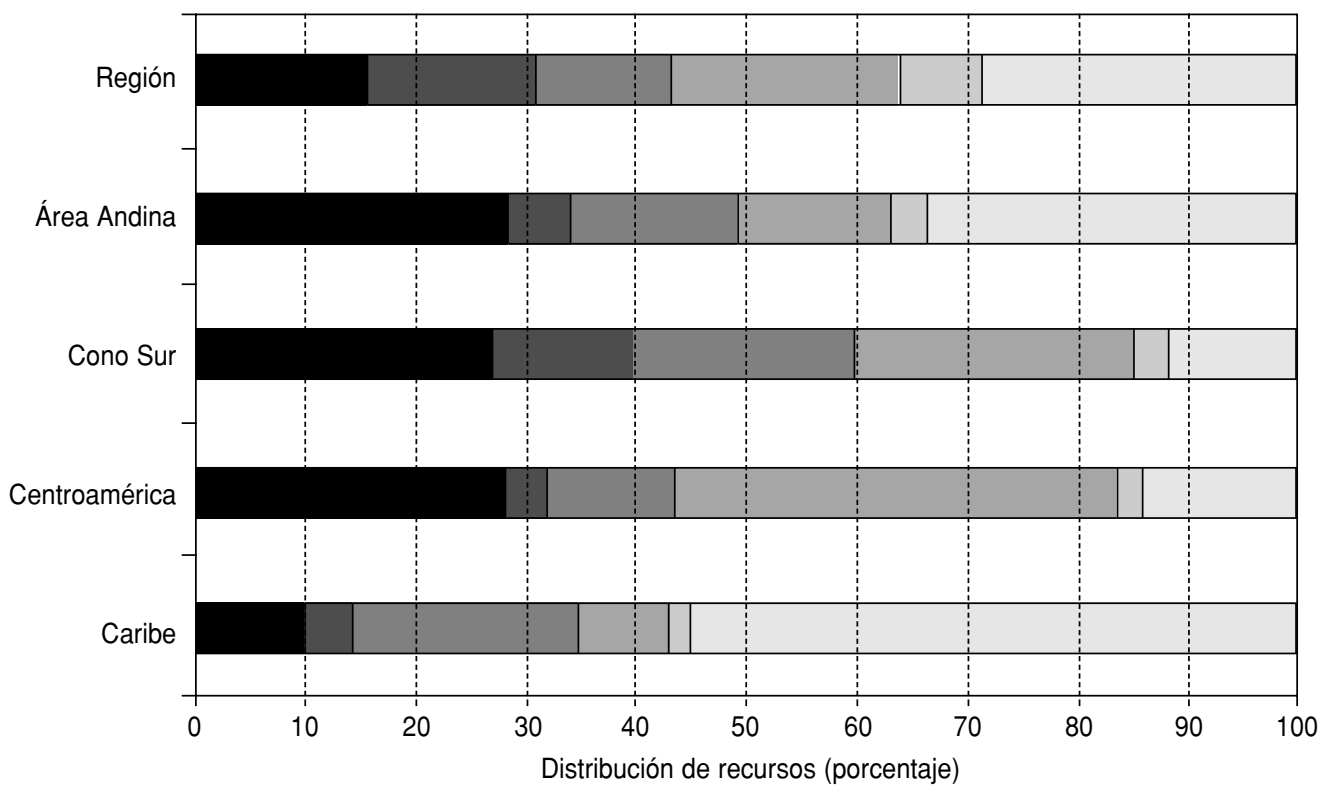

Movilización de recursos

Formulación de normas, planes y políticas

Diseminación de información

Fomento de la investigación
Formación de personal

Asesoramiento técnico directo fomento de la cooperación entre sectores, países y organismos; determinación de acciones entre partes interesadas; cooperación con empresas nacionales; expansión del sector privado; cooperación internacional y definición de los resultados de las negociaciones

2. Sistemas de vigilancia e información

Establecimiento de sistemas de información para la vigilancia, toma de decisiones, seguimiento $\mathrm{u}$ otras acciones en materia de salud; sistemas de monitoreo y de vigilancia; sistemas de control de calidad; sistemas y centros de documentación, bibliotecas de referencia y bases de datos; sistemas de garantía de la calidad; redes de información, y extensiones del Internet

3. Normas y pautas

Identificación y suministro de información sobre normas que regulen las acciones en pro de la salud en el ámbito nacional o internacional; material informativo (por ejemplo, manuales, series de referencia, pautas, reglamentos sectoriales, programas de estudios, etc.); leyes sectoriales, reglamentos, libros, especificaciones de garantía de la calidad, indicadores

4. Estudios de investigación y evaluación

Generación de información nueva mediante el análisis, la investigación y la revisión de temas y mediante actividades de evaluación, valoración y examen
5. Planes, proyectos y políticas

Elaboración de proyectos, estrategias y planes de acción para los servicios, programas, instituciones y centros; proyectos para obtener financiamiento externo; propuestas de políticas y planes detallados

6. Métodos, modelos y tecnologías

Implantación de métodos y procesos para mejorar la calidad, eficacia y eficiencia de los programas, instituciones y sistemas de salud y de los procesos tecnológicos y las tecnologías

7. Programas de capacitación

Desarrollo de recursos humanos y de programas de capacitación, particularmente en los países

8. Campañas promocionales y cabildeo

Celebración de eventos y campañas para la toma de conciencia

9. Apoyo directo

Compra y transferencia de equipo, vacunas, materiales biológicos y suministros para los países; asignación de recursos humanos y financieros; contratación de personal; aporte de suplementos salariales locales

En la segunda fase del estudio se analizaron la validez y utilidad de la clasificación propuesta. La Oficina de Análisis y Planificación Estratégica (Division of Analysis and Strategic Planning, DAP) de 
la OPS convocó a 70 funcionarios de cinco oficinas de país y de dos divisiones regionales a participar en grupos de consulta. El criterio de selección de las unidades se basó en la representación equilibrada de las áreas geográficas y programáticas y dependió de la disponibilidad de las unidades para realizar la consulta durante un período de 2 meses, ya que el estudio debía concluirse con tiempo suficiente para modificar las guías de elaboración del BPB de 2000-2001.

Los grupos de consulta trabajaron con los siguientes objetivos:

- reformular los resultados esperados del BPB de 1998-1999 utilizando la nueva clasificación de resultados esperados funcionales;

- proponer nuevas categorías de resultados esperados para los que no se pudieron reformular;

- hacer comentarios y sugerencias sobre la validez y utilidad de la clasificación propuesta.

Se estudiaron 92 resultados esperados de proyectos en los países y 37 resultados esperados de proyectos regionales para observar si contribuían al propósito del proyecto de cooperación técnica y si su logro dependía de la Secretaría. A continuación, los funcionarios responsables de los proyectos determinaron si los resultados respondían a la nueva clasificación y, en caso contrario, los reformularon utilizando una o más de las categorías incluidas en ella (cuadro 1). Los hallazgos más importantes de los grupos de consulta fueron los siguientes:

\section{Reformulación de los resultados esperados}

En la mayoría de los casos, los funcionarios responsables de los resultados esperados tuvieron que aclarar cuál era el compromiso adquirido por la OPS en el proyecto, pues los demás participantes no podían identificarlo mediante la simple lectura.

La mayoría de los 129 resultados esperados analizados pudieron reformularse y desagregarse según la clasificación propuesta. De ese modo se obtuvieron 281 resultados. El uso de la clasificación propuesta obligó a describir resultados esperados más concretos que los originales $\mathrm{y}$, por consiguiente, la distribución de resultados esperados en el caso de los países fue más equilibrada; en el nivel regional, se observó un mayor número de resultados esperados en la categoría de planes, proyectos y políticas, y de métodos, modelos y tecnologías. La nueva distribución de los resultados esperados refleja las diferentes funciones que desempeña la OPS en los países y en el ámbito regional (figura 4).

\section{Resultados esperados propuestos}

Aunque algunos grupos sugirieron agregar las categorías de diseminación de información, mo-
CUADRO 1. Distribución de resultados esperados en los países y en la Región, según la nueva clasificación propuesta

\begin{tabular}{|c|c|c|c|c|}
\hline \multirow{2}{*}{$\begin{array}{c}\text { Categorías de } \\
\text { resultados esperados }\end{array}$} & \multicolumn{2}{|c|}{ Países } & \multicolumn{2}{|c|}{ Región } \\
\hline & No. & $\%$ & No. & $\%$ \\
\hline 1. Redes de cooperación y & & & & \\
\hline alianzas & 23 & 10,4 & 2 & \\
\hline 2. Sistemas de vigilancia e & & & & \\
\hline información & 19 & 8,6 & 1 & \\
\hline 3. Normas y pautas & 20 & 9,0 & 4 & \\
\hline 4. Estudios de investigación & & & & \\
\hline y evaluación & 30 & 13,5 & 3 & \\
\hline 5. Planes, proyectos y políticas ${ }^{a}$ & 24 & 10,9 & 28 & \\
\hline 6. Métodos, modelos y tecnologías & 22 & 10,0 & 12 & \\
\hline 7. Programas de capacitación & 48 & 21,7 & 5 & \\
\hline 8. Campañas promocionales y & & & & \\
\hline cabildeo & 13 & 5,9 & 1 & \\
\hline 9. Apoyo directob & 16 & 7,0 & 0 & \\
\hline 10. Otros ${ }^{c}$ & 7 & 3,0 & 3 & \\
\hline Total & 222 & 100 & 59 & 10 \\
\hline
\end{tabular}

${ }^{a}$ Abarca cuatro resultados esperados que previamente se clasificaban como movilización de recursos.

${ }^{b}$ Abarca nueve resultados esperados que previamente se clasificaban como asesoramiento técnico directo.

${ }^{c}$ Abarca seis resultados esperados que previamente se clasificaban como diseminación de información.

vilización de recursos, y asesoramiento técnico directo a la clasificación de resultados esperados propuesta, fue difícil asignarles indicadores por no tratarse de productos, sino de modalidades de trabajo que utiliza la OPS para producir los resultados esperados. El cuadro 2 muestra ejemplos de resultados esperados tomados del BPB de 1998-1999 y reformulados posteriormente según la clasificación propuesta. La diseminación de información se incorporó a la categoría "Otros" en los BPB de tres países y de tres regiones; la movilización de recursos se incorporó a la categoría de planes, proyectos y políticas de cuatro BPB regionales y el asesoramiento técnico directo se incorporó a la categoría de apoyo directo en los BPB de nueve países.

\section{Comentarios y sugerencias}

Los grupos coincidieron en que la clasificación de los resultados esperados propuesta reflejaba los productos de los proyectos de cooperación técnica y recomendaron su uso para definir con mayor precisión esos resultados y facilitar el proceso de elaboración de los proyectos y la negociación con los países.

También señalaron que algunas actividades de cooperación técnica no pueden identificarse como productos tangibles ni se registran en los proyectos del AMPES. Constituye un ejemplo el tiempo que dedican los funcionarios a respaldar los procesos y actividades (participación en reunio- 


\section{FIGURA 4. Resultados esperados reformulados según la clasificación propuesta}

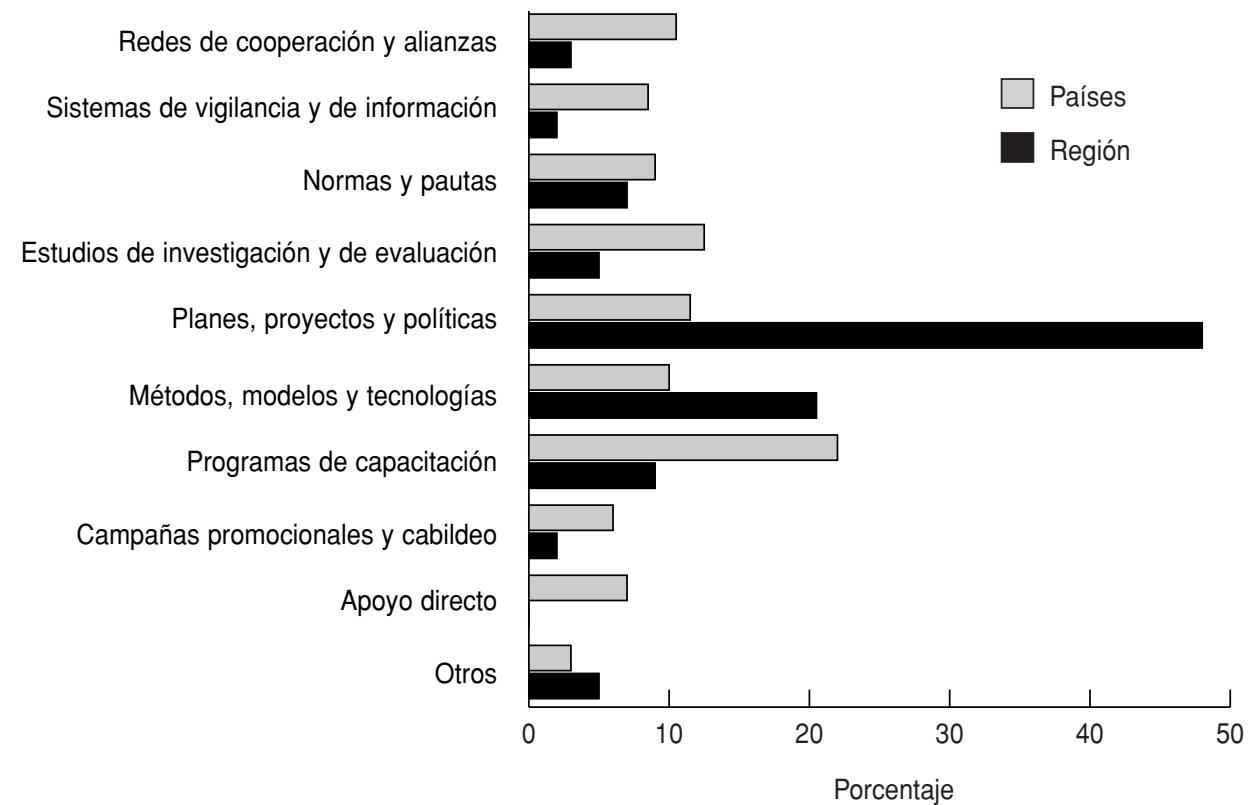

CUADRO 2. Reclasificación de los resultados esperados en el presupuesto bienal por programas (PBP) de 1998-1999

\begin{tabular}{|c|c|}
\hline Resultados esperados & Resultados esperados reclasificados ${ }^{a}$ \\
\hline $\begin{array}{l}\text { Ampliación de la cobertura y representatividad del sistema estadístico de } \\
\text { registro de daños a la salud (morbilidad y mortalidad) en los niveles } \\
\text { nacional y regional }\end{array}$ & $\begin{array}{l}\text { (7) Capacitación del personal de los sistemas estadísticos } \\
\text { para el registro de daños a fin de que incorporen la } \\
\text { Clasificación Estadística de Enfermedades en el sistema } \\
\text { estadístico }\end{array}$ \\
\hline $\begin{array}{l}\text { Mejoramiento de la orientación de las políticas públicas, los servicios y las } \\
\text { intervenciones mediante metodologías de ejecución y evaluación per- } \\
\text { manentes del análisis de la situación de salud en los niveles subregio- } \\
\text { nal, nacional y local }\end{array}$ & $\begin{array}{l}\text { (6) Elaboración de métodos de análisis de la situación de } \\
\text { salud durante el proceso de capacitación en el servicio }\end{array}$ \\
\hline $\begin{array}{l}\text { Fortalecimiento del sistema de vigilancia epidemiológica para la notifica- } \\
\text { ción oportuna de los casos de sarampión, tétanos neonatal, PFA y } \\
\text { hepatitis B }\end{array}$ & $\begin{array}{l}\text { (3) Distribución de normas y pautas internacionales para } \\
\text { evaluar el sistema de vigilancia }\end{array}$ \\
\hline $\begin{array}{l}\text { Fortalecimiento de la iniciativa de comunidades saludables en el nivel } \\
\text { nacional }\end{array}$ & $\begin{array}{l}\text { (1) Establecimiento de redes de cooperación de munici- } \\
\text { pios saludables } \\
\text { (6) Elaboración participatoria de métodos para la planifica- } \\
\text { ción de base }\end{array}$ \\
\hline $\begin{array}{l}\text { Fortalecimiento de la capacidad gerencial del personal de salud de los } \\
\text { ministerios de salud, a fin de llevar a cabo el proceso de cambio geren- } \\
\text { cial necesario para la reforma del sector de la salud }\end{array}$ & $\begin{array}{l}\text { (7) Capacitación gerencial del personal de salud } \\
\text { (5) Desarrollo de un plan de mercadeo para mejorar la } \\
\text { imagen del Ministerio de Salud } \\
\text { (1) Mantenimiento de las redes de cooperación entre pro- } \\
\text { fesionales coordinadas por los ministerios nacionales y } \\
\text { las autoridades regionales de salud }\end{array}$ \\
\hline $\begin{array}{l}\text { Promoción de la ejecución, seguimiento y evaluación de las políticas y } \\
\text { programas de salud reproductiva }\end{array}$ & $\begin{array}{l}\text { (4) Realización y revisión de estudios sobre la gerencia } \\
\text { (2) Establecimiento y mantenimiento de sistemas de } \\
\text { información sobre la salud reproductiva } \\
\text { (7) Capacitación de profesionales de salud } \\
\text { (1) Creación de redes de cooperación de recursos huma- } \\
\text { nos en salud }\end{array}$ \\
\hline
\end{tabular}

a Los números entre paréntesis corresponden al número de la categoría en la nueva clasificación.

nes, aportes para la discusión de grupos de trabajo, llamadas telefónicas, etc.). Los participantes consideraron que debían establecerse resultados esperados con sus indicadores para facilitar la descripción y valoración de ese aspecto del trabajo en el contexto del proceso de evaluación de los profesionales.

En los casos en que la OPS apoya una iniciativa nacional pero no tiene bajo su responsabilidad exclusiva los resultados esperados (identificados 
como productos de la cooperación técnica), los proyectos de cooperación técnica deberán identificar los resultados de los cuales la Secretaría se hace responsable junto con el país.

\section{CONCLUSIONES}

La identificación de resultados esperados permite definir con mayor precisión los productos de los proyectos de cooperación técnica de la OPS, el grado de responsabilidad que tiene la Organización y las bases para estimar los recursos y facilitar el seguimiento y la evaluación. Como consecuencia inmediata de los hallazgos de las dos primeras fases del estudio aquí descrito sobre los enfoques funcionales, se incorporó la clasificación de resultados esperados propuesta por los grupos de consulta en las guías de preparación del BPB de 2000-2001 de todas las unidades. Asimismo, ya se ha diseñado y comenzado una tercera etapa de análisis para evaluar el efecto de la cooperación técnica a partir de las modificaciones incorporadas en la programación de las actividades

\section{SYNOPSIS}

\section{In search of the results of technical cooperation}

For the Pan American Health Organization (PAHO), technical cooperation (TC) is the process by which the PAHO Member States work with the Organization, as equal partners, to identify and reach their own health goals and to promote self-sufficiency in health development, through programs that respond to those countries' needs and national priorities. Since 1978, PAHO has used the American Re- gional Planning, Programming, Monitoring, and Evaluation System (AMPES) to establish management procedures and to facilitate decision-making in health TC. As part of AMPES, PAHO uses a "logical approach to project management" to structure the work program of the Organization and to identify the expected results from TC activities and TC resource investments. This project management approach, which replaces the "functional approaches" system used to date, also helps establish a causative relationship between the programmed activities and the results that $P A H O$ expects, and between the activities and the hoped-for outcomes in the countries.

As part of an ongoing process of rethinking international health TC, several years ago PAHO began a four-phase study on the usefulness and validity of functional approaches and on the need to propose new ones or to modify existing ones. The results of the initial phase showed it was difficult to classify the activities because the functionalapproaches categories were not mutually exclusive and the TC activities were complex. Further, the expected results did not specify the product for which the PAHO Secretariat was accountable within a certain time frame nor the Secretariat's level of responsibility. Thus, a new and more flexible classification of expected results was proposed, with the following categories: cooperation networks and alliances; surveillance and information systems; standards and guidelines; research and evaluation studies; plans, projects, and policies; methods, models, and technologies; training programs; promotional campaigns and advocacy; and direct support. In the second phase of the study, it was concluded that the proposed classification system made it possible to more precisely identify the products of PAHO technical cooperation projects, the Organization's degree of responsibility, and the bases for estimating needed resources. The new system could also facilitate monitoring and evaluation. In addition, the third phase of analyzing the functional approaches has begun. Its objective is to evaluate the effect of technical cooperation based on the changes incorporated in the programming of activities.

\section{REFERENCIAS}

1. Organización Panamericana de la Salud. Orientaciones Estratégicas y Programáticas 1995-1998. Washington, DC: OPS 1995. (Documento oficial 269).

2. Organización Mundial de la Salud. Noveno programa general de trabajo para el período 1996-2001. Ginebra: OMS; 1994. (Serie Salud para Todos 11).

3. Organización Panamericana de la Salud, Oficina de Análisis y Planificación Estratégica. Sistema de Planificación, Programación y Evaluación de la Cooperación Técnica (AMPES). Washington, DC: OPS; 1998. (OPS/DAP/98.5.38).
4. Organización Panamericana de la Salud, Oficina de Análisis y Planificación Estratégica. Enfoque lógico para la gestión de proyectos en la OPS. Washington, DC: OPS; 1996. (Informe técnico OPS/DAP/96.1.18).

5. Alleyne GAO. Hacia una taxonomía de la cooperación técnica en salud: informe especial. Bol Oficina Sanit Panam 1992;112 (1):1-11.

6. Organización Panamericana de la Salud, Oficina de Análisis y Planificación Estratégica. Replanteamiento de la Cooperación Técnica Internacional en Salud (RITCH): informe final de un seminario internacional celebrado del 27 al 29 de noviembre de 1995 . Washington, DC: OPS; 1996. (OPS/DAP/96.4.24).

7. Organización Panamericana de la Salud. Presupuesto por programas 1998-1999: Organización Panamericana de la Salud, propuesta 1998-1999; Organización Mundial de la Salud, Región de las Américas, 1998-1999; Organización Panamericana de la Salud, Anteproyecto 2000-2001; Organización Mundial de la Salud, Región de las Américas, Anteproyecto 2000-2001. Washington, DC: OPS; 1997. (Documento oficial 281). 